\title{
Identification of Biomarkers in Patients with Thrombotic Thrombocytopenic Purpura Presenting with Large and Small Ischemic Stroke
}

\author{
Chen Lin $^{a}$ Raima Memon ${ }^{b} \quad$ Jingrui Sui ${ }^{b} \quad$ X. Long Zheng ${ }^{b, c}$ \\ a Department of Neurology, The University of Alabama at Birmingham, Birmingham, AL, USA; ${ }^{b}$ Department of \\ Pathology, The University of Alabama at Birmingham, Birmingham, AL, USA; ${ }^{C}$ Department of Pathology and \\ Laboratory Medicine, University of Kansas Medical Center, Kansas City, KS, USA
}

\section{Keywords}

Stroke etiology $\cdot$ Neuroimaging of stroke $\cdot$ Thrombotic thrombocytopenic purpura $\cdot$ ADAMTS13 $\cdot$ Neutrophil extracellular trap · Inflammation

\begin{abstract}
Background: Thrombotic thrombocytopenic purpura (TTP) is a rare blood disorder resulting in organ damage including ischemic strokes. We sought to characterize the neuroimaging patterns of stroke in a large cohort of patients with immune-mediated TTP (iTTP) and determined their associations with clinical and laboratory parameters and outcomes. Methods: We analyzed the Alabama TTP Registry who had laboratory confirmation of acute iTTP. We reviewed the neuroimaging patterns of those with ischemic stroke on MRI, clinical information, and laboratory results. Small ischemic strokes were $\leq 20 \mathrm{~mm}$ in their maximum diameter in the axial plane. Large ischemic strokes were $>20 \mathrm{~mm}$. Student $t$ test, Mann-Whitney $\mathrm{U}$ test, and $\mathrm{X}^{2}$ test were all used for data analysis. Results: Of 108 iTTP patients, 21 had ischemic stroke on neuroimaging. The median platelet count in these patients was $12 \times 10^{9} / \mathrm{L}$ (interquartile range, IQR, $8.8-21 \times$ $10^{9} / \mathrm{L}$ ), plasma ADAMTS13 activity $1.8 \mathrm{U} / \mathrm{dL}$ (IQR 0-4.5 U/dL), and the mean plasma level of anti-ADAMTS13 IgG was 6,595.8 U/mL (SD 3,448.9 U/mL). Comparison between patients with large ischemic strokes $(n=10)$ and small ischemic
\end{abstract}

karger@karger.com www.karger.com/cee

Karger"

BOPEN ACCESS
(C) 2021 The Author(s)

Published by S. Karger AG, Basel

This article is licensed under the Creative Commons AttributionNonCommercial-NoDerivatives 4.0 International License (CC BYNC-ND) (http://www.karger.com/Services/OpenAccessLicense). Usage and distribution for commercial purposes as well as any distribution of modified material requires written permission. strokes $(n=11)$ revealed that patients with small stroke were older $(p=0.043)$ and had higher plasma levels of citrullinated histone $3(p=0.006)$ and histone/DNA complex $(p=0.014)$ than those with large strokes. There were no significant differences between 2 stroke groups in mortality or exacerbation. Conclusions: iTTP patients can present with large ischemic strokes and are usually younger. Further research should be performed in assessing different etiologies of iTTP-associated stroke based on neutrophil extracellular trap formation biomarkers (e.g., histone markers) seen in small ischemic stroke.

(c) 2021 The Author(s)

Published by S. Karger AG, Basel

\section{Introduction}

Thrombotic thrombocytopenic purpura (TTP) is a rare blood disorder, leading to multisystem organ damage and mortality $[1,2]$. The incidence ranges from 3 to 13 cases per million residents per year $[1,3]$. The pathophysiology of immune-mediated TTP (iTTP) is the result of an acquired inhibition of ADATMS13 activity due to autoantibodies against ADAMTS13 $[4,5]$, while con-

Chen Lin

Department of Neurology

The University of Alabama at Birmingham, RWUH M226

1813 6th Avenue South, Birmingham, AL 35294 (USA)

clin@uabmc.edu

\section{Long Zheng}

Department of Pathology and Laboratory Medicine

The University of Kansas Medical Center

3901 Rainbow Blvd, 5016 Delp, Kansas City, KS 66160 (USA)

xzheng2@ kumc.edu 
genital TTP is caused by an inherited deficiency of ADAMTS13 due to mutations in ADAMTS13 [6-8]. ADAMTS13 is a plasma metalloproteinase responsible for cleaving ultra-large von Willebrand factor, released from endothelial cells $[9,10]$. A reduction of plasma ADAMTS13 activity leads to an accumulation of highly adhesive ultra-large von Willebrand factor multimers on endothelial surface $[9,10]$ and in circulation [11-13]. This ultra-large von Willebrand factor captures flowing platelets in circulation, resulting in excessive thrombus formation in areas of endothelial activation and/or injury [14-16]. If left unrecognized or untreated, TTP carries an exceedingly high mortality (90-95\%) [1, 2, 17]. Therapeutic plasma exchange (TPE) or plasma infusion has dramatically reduced mortality and morbidity rates $[2$, $14,17]$. Recent advances in therapeutic strategy including the use of rituximab [18-20] and caplacizumab [21-23] have further improved survival rate and long-term outcome.

The typical presentation of TTP incudes thrombocytopenia, microangiopathic hemolytic anemia, and various degrees of organ injury $[2,24]$. At initial diagnosis, roughly half of patients report neurological symptoms, but ultimately $90 \%$ of TTP patients develop some sort of central nervous system involvement $[25,26]$. The most common symptoms include fluctuating mental status or persistent encephalopathy, aphasia, paresthesia, motor deficits, and seizure [27]. The presumptive mechanism is from thrombi in small vessels that lead to transient occlusion or small infarcts [14]. The typical small ischemic strokes seen in TTP are thought to be largely reversible in most patients if they survive the systemic issues related to TTP [25]. Large ischemic strokes are thought to be very rare neurological presentations in TTP. The literature on stroke in TTP is limited to only case reports or small series $[14,26,28-31]$. To our knowledge, a comparison of stroke neuroimaging findings and its association with laboratory values and clinical outcomes has never been reported. In this study, we sought to carefully characterize the neuroimaging patterns of stroke in a large cohort of patients with TTP and determined their associations with patient clinical characteristics, laboratory values, and clinical outcomes.

\section{Methods}

\section{Patient Selection and Data Collection}

The study protocol was approved by the International Review Committee at the University of Alabama at Birmingham (UAB). All the patients with suspected TTP (presenting with one or more of the diagnostic criteria for TTP mentioned below) who were referred to the UAB Apheresis service and captured in the Alabama TTP Registry between the period April 2006 and December 2019 for TPE were included in this study. A total of 109 patients were diagnosed with acute TTP. Of these patients, we reviewed whether they had complaints of neurological symptoms (i.e., headache, slurring of speech, forgetfulness, dizziness, visual changes, numbness or weakness of the extremities, coma or seizures) and whether neuroimaging was available to confirm ischemic stroke. Three patients were excluded because they were transferred from outside hospitals and imaging was not available for the review. While the Registry contains both congenital TTP $(n=1)$ and iTTP $(n=108)$, only iTTP patients who had ischemic strokes were included in the analysis. TTP was determined to be the etiology of all strokes included in the analysis.

The diagnostic criteria for TTP in this study were the following: severe thrombocytopenia and microangiopathic hemolytic anemia (e.g., low hemoglobin, low hematocrit, elevated lactate dehydrogenase, and presence of schistocytes on peripheral blood smear), with or without evidence of end-organ damage, including fluctuating neurological symptoms and fever or renal impairment $[20,32]$. Plasma ADAMTS13 activity were all $<5$ IU/dL with inhibitor $>0.4 \mathrm{U} / \mathrm{mL}$, and/or anti-ADAMTS13 IgG $>15 \mathrm{U} / \mathrm{mL}$ [33]. Patient clinical and routine laboratory information was extracted from the electronic medical record and Alabama TTP registry database.

\section{Neuroimaging Studies and Stroke Classification}

Neuroimaging studies performed at the time of presentation for stroke or transient ischemic attack were included for analysis. The magnetic resonance imaging (MRI) was performed using the UAB stroke protocol. MRI was done using a Philips 1.5-T scanner. Cerebral MRI was done including T1- and T2weighted sequences, diffusion-weighted imaging (DWI), and FLAIR. The scan was done with a slice thickness of $6 \mathrm{~mm}$ and at a slice interval of $1 \mathrm{~mm}$ with DWI b-value $=0,1,000 \mathrm{~s} / \mathrm{mm}^{2}$. All neuroimaging studies were independently reviewed by 2 of the investigators (C.L. and R.M.) to verify imaging results. One of the investigators (C.L.) was blinded to the clinical data. Radiological changes were categorized by large or small ischemic strokes if they exhibited a high-intensity signal on DWI and a corresponding low diffusion coefficient on the apparent diffusion coefficient map. The infarcts were classified into small or large ischemic strokes. Small ischemic strokes were classified by the Standards for Reporting Vascular changes on Neuroimaging (STRIVE) position statement on small vessel disease [34]. We considered small ischemic strokes to be $\leq 20 \mathrm{~mm}$ in their maximum diameter in the axial plane [34]. Large ischemic strokes were $>20 \mathrm{~mm}$. We further differentiated small strokes as being territorial (cortical), lacunar (deep), or a combination of both $[35,36]$. By consensus, clinical and radiographic data were reviewed to determine Trial of Org 10172 in Acute Stroke Treatment (TOAST) subtype for each confirmed case $[37,38]$.

\section{Laboratory Assessment}

Routine and special laboratory analyses were performed on the initial blood samples as part of patient care, including complete blood count, creatinine, prothrombin time, partial thromboplastin time, fibrinogen, D-dimer, lactate dehydrogenase, troponin, and so on. These data were available from patients' electronic medical
30

Cerebrovasc Dis Extra 2021;11:29-36 DOI: $10.1159 / 000513574$
Lin/Memon/Sui/Zheng 
Table 1. Demographic features of TTP patients with neuroimaging $(n=21)$

\begin{tabular}{lc}
\hline Parameters & Values \\
\hline Age, years & $49.9 \pm 10.9$ \\
Gender, $n(\%)$ & $12(57.1)$ \\
$\quad$ Female & $9(42.9)$ \\
Male & $15(71.4)$ \\
Race, $n(\%)$ & $5(23.8)$ \\
Black & $1(4.8)$ \\
White & \\
Hispanic & $14(66.7)$ \\
Disease status, $n(\%)$ & $7(33.3)$ \\
Initial & \\
Relapse & $10(47.6)$ \\
Blood group, $n(\%)$ & $11(52.4)$ \\
O & \\
Non-O & $16(76.2)$ \\
Comorbidities, $n(\%)$ & $3(14.3)$ \\
Hypertension & \\
Diabetes mellitus & $3(14.3)$ \\
Symptoms, $n(\%)$ & $8(38.1)$ \\
Fever & $2(9.5)$ \\
Chest pain & \\
Abdominal pain &
\end{tabular}

Age is expressed as mean $\pm \mathrm{SD}$. All other parameters are expressed as number and percentage of patients. TTP, thrombotic thrombocytopenic purpura; O, blood type $\mathrm{O}$.

records [39]. Additionally, all patients with TTP had blood drawn at the time of presentation for investigational assays. In this case, whole-blood samples were collected from an apheresis catheter before the initiation of TPE and anticoagulated with $0.39 \%$ sodium citrate. After being centrifuged $(1,500 \times g$ for $15 \mathrm{~min})$ within $2 \mathrm{~h}$ of collection, platelet-poor plasma was separated from the cellular components, aliquoted, and stored at $-80^{\circ} \mathrm{C}$ until assays.

Plasma ADAMTS13 activity, inhibitor, and anti-ADAMTS13 IgG were determined in a reference laboratory (Versiti, Milwaukee, WI) and in-house assays as previously described [33].

\section{Plasma Histone DNA Complex, Cell-Free DNA, and}

Citrullinated Histone

Plasma levels of histone/DNA complex (Millipore Sigma) and citrullinated histone $\mathrm{H}_{3}$ (Cayman Chemicals, Ann Arbor, MI, USA) were determined by the commercial ELISA kits according to the manufacturers' instructions. Cell-free DNA was determined using the Quant-IT PicoGreen dsDNA assay (Thermo Fisher Scientific) according to the manufacturer's instructions.

\section{Statistical Analysis}

Means, median, and percentage of ordinal variables were calculated using SPSS software. Student $t$ test and Mann-Whitney U test or $\chi^{2}$ test was used for data with normal distribution and categorical data, respectively. A $p$ value $<0.05$ and 0.01 was statistically significant and highly significant, respectively.

Biomarker and Stroke Size in iTTP
Table 2. Presenting laboratory parameters of iTTP patients with neuroimaging $(n=21)$

\begin{tabular}{ll}
\hline Parameters & Values \\
\hline Routine laboratory tests & \\
White blood cells, $\times 10^{9} / \mathrm{L}$ & $10.9 \pm 4.4$ \\
Hemoglobin, g/dL & $8.7 \pm 1.9$ \\
Hematocrit, $\%$ & $25.5 \pm 5.2$ \\
Platelet count, $\times 10^{9} / \mathrm{L}$ & $12.0(8.8-21.0)$ \\
Lactate dehydrogenase, U/L & $963.6 \pm 466.9$ \\
Creatinine, mg/dL & $1.4(0.90-1.68)$ \\
Prothrombin time, s & $14.85(14-16.1)$ \\
Partial thromboplastin time, s & $32.0 \pm 6.5$ \\
Fibrinogen, mg/dL & $420.6 \pm 108.6$ \\
D-dimer, ng/mL $(n=16)$ & $1,369.5(711.8-2,920.5)$ \\
Troponin, ng/mL & $0.6(0.2-2.2)$ \\
Special laboratory tests & $<5.0$ \\
ADAMTS13 activity, U/dL & \\
Anti-ADAMTS13 IgG, U/mL & $6,595.8 \pm 3,448.9$ \\
$\quad(n=17)$ & $1.4(0.6-3.2)$ \\
ADAMTS13 inhibitor, BU $(n=15)$ & \\
Citrullinated histone $\mathrm{H}_{3}, \mathrm{ng} / \mathrm{mL}$ & $3.6(3.1-7.1)$ \\
$\quad(n=15)$ & $71.6(31.3-171.2)$ \\
Histone/DNA complex, U/mL & $987.6(818.0-1,195.5)$ \\
$\quad(n=18)$ &
\end{tabular}

White blood cells, hemoglobin, hematocrit, lactate dehydrogenase, partial thromboplastin time, fibrinogen, and antiADAMTS13 IgG are expressed as the mean \pm SD. All other parameters are expressed as the median and interquartile range. TTP, thrombotic thrombocytopenic purpura; BU, Bethesda unit.

\section{Results}

Clinical and Laboratory Characteristics of the Patients with Reviewable Neuroimaging in the Alabama TTP Cohort

Out of 108 iTTP patients in our Alabama Registry, 21 of the iTTP patients had ischemic stroke on neuroimaging. Of these, the mean age was 49.9 (SD 10.9) years at the time of admission. Twelve (57.1\%) patients were female and $9(42.9 \%)$ were male. Fifteen $(71.4 \%)$ of these patients were African Americans. Fourteen (66.7\%) were initial and $7(33.3 \%)$ were relapsed presentations of iTTP. In this cohort, blood group O patients comprised $47.6 \%$ of the patients. The majority of the patients had comorbidities, including hypertension (76.2\%) and diabetes mellitus (14.3\%). All the patients had neurological symptoms, $14.3 \%$ had fever, $38.1 \%$ had chest pain, and $9.5 \%$ had abdominal pain (Table 1). 

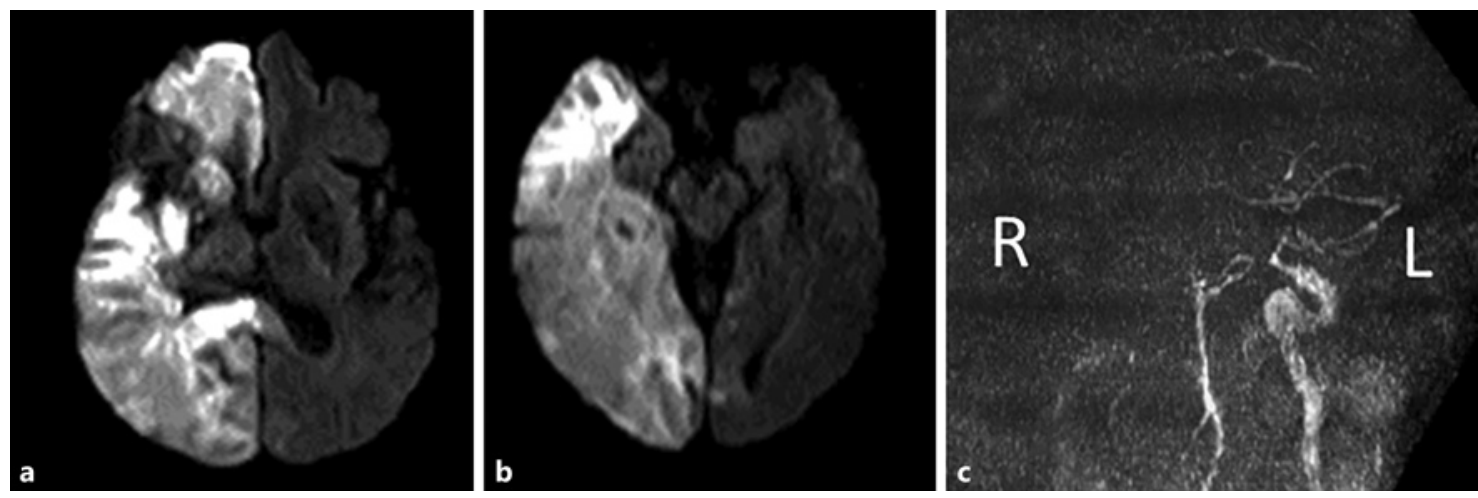

Fig. 1. Example of large territorial infarct with diffusion restriction of the anterior cerebral and middle cerebral arteries (a) and middle cerebral and posterior cerebral arteries (b). c Magnetic resonance angiography in the same patient with complete occlusion of the right internal carotid and posterior cerebral arteries.

Table 3. Treatment and outcomes of iTTP patients with neuroimaging $(n=21)$

\begin{tabular}{ll}
\hline Parameters & Values \\
\hline Who received TPE & $21(100)$ \\
Who received rituximab & $14(66.7)$ \\
Outcome & \\
Death & $4(19.1)$ \\
Remission & $7(33.3)$ \\
Recurrence & $9(42.9)$ \\
Exacerbation & $7(33.3)$ \\
Relapse & $2(9.5)$ \\
\hline
\end{tabular}

All the parameters are expressed as number and percentage of patients. TPE, therapeutic plasma exchange; $n$, total number of patients with imaging.

Of the 21 patients, 17 had "other" as the TOAST subtype, attributed directly to TTP. Four patients are considered cryptogenic because more than 1 etiology could have contributed to their stroke but all 4 were noted to have TTP as one of those contributing etiologies. Seventeen patients had neurology formally consulted in the hospital though all eventually followed with a neurologist. During index hospitalization for the stroke, $10 \mathrm{had}$ extracranial carotid duplex ultrasonography. Nineteen had a transthoracic echocardiogram, 7 had transesophageal echocardiogram, and 13 had either CT or MR angiography. Of the 10 patients with small strokes, we identified 7 cortical, 3 mixed, and no patients with only lacunar infarcts.
Laboratory parameters in all 21 iTTP patients at the time of admission are shown in Table 2. The laboratory results fully supported the diagnosis of iTTP, with the median platelet count $12 \times 10^{9} / \mathrm{L}$ (interquartile range, IQR, 8.8-21 $\times 10^{9} / \mathrm{L}$ ) and plasma ADAMTS13 activity 1.8 $\mathrm{U} / \mathrm{dL}$ (IQR 0-4.5 U/dL). The mean hematocrit was $25.5 \%$ (SD 5.2\%) and lactate dehydrogenase 963.6 U/L (SD 466.9 $\mathrm{U} / \mathrm{L})$. The mean plasma level of anti-ADAMTS13 IgG was $6,595.8 \mathrm{U} / \mathrm{mL}$ (SD 3,448.9 U/mL). All 21 patients received TPE plus corticosteroid therapy, and $14(66.7 \%)$ were treated with rituximab. Of 21 patients, 4 (19.1\%) did not survive from the acute episode, 8 (38.1\%) were followed by sustained remission, and $9(42.9 \%)$ exhibited a disease recurrence (Table 3 ).

\section{Correlations between Ischemic Stroke Size and \\ Demographic and Laboratory Parameters in iTTP \\ Patients}

Based on the criteria we described in the method, the infarcts of the 21 iTTP patients were classified into small $(n=10)$ and large $(n=11)$ ischemic strokes. The correlations between stroke size and demographics, clinical presentation, laboratory parameters, and patient outcomes were determined. Compared with patients with large ischemic strokes, those with small ischemic strokes were much older $(p=0.043)$ and had higher levels of citrullinated histone $3(p=0.006)$ and histone/DNA complex $(p=0.014$; Table 4$)$. Using the log-rank test, we determined the size of the ischemic stroke and the outcome of the patients. Patients with small ischemic stroke did not have a higher risk of death than those with large ischemic stroke (hazard ratio, HR 1.17, 95\% CI 0.16-8.36; $p=0.88$; Table 4). It looked like that patients with small ischemic 
Table 4. Association between the stroke size and demographic or laboratory parameters in patients with iTTP $(N=21)$

\begin{tabular}{|c|c|c|c|}
\hline Variables & Large $(n=11)$ & Small $(n=10)$ & $p$ value \\
\hline \multicolumn{4}{|l|}{ Demographics } \\
\hline Age $($ mean $\pm S D)$, years & $45.4 \pm 11.2$ & $54.8 \pm 8.4$ & $0.043^{\mathrm{a}}$ \\
\hline Female, $n(\%)$ & $6(54.5)$ & $6(60)$ & $0.58^{\mathrm{b}}$ \\
\hline African American, $n(\%)$ & $7(63.6)$ & $7(70)$ & $1.00^{\mathrm{b}}$ \\
\hline Disease status, initial, $n(\%)$ & $6(54.5)$ & $8(80)$ & $0.22^{\mathrm{b}}$ \\
\hline Blood group $\mathrm{O}, n(\%)$ & $4(36.4)$ & $6(60)$ & $0.26^{\mathrm{b}}$ \\
\hline \multicolumn{4}{|l|}{ Comorbidities } \\
\hline Hypertension, $n(\%)$ & $9(81.8)$ & $7(70)$ & $0.64^{\mathrm{b}}$ \\
\hline Diabetes mellitus, $n(\%)$ & $3(27.3)$ & $0(0)$ & $0.12^{\mathrm{b}}$ \\
\hline Fever, $n(\%)$ & $2(18.2)$ & $1(10)$ & $1.00^{\mathrm{a}}$ \\
\hline Chest pain, $n(\%)$ & $3(27.3)$ & $5(50)$ & $0.39^{\mathrm{b}}$ \\
\hline Abdominal pain, $n(\%)$ & $1(9.1)$ & $1(10)$ & $1.00^{\mathrm{b}}$ \\
\hline \multicolumn{4}{|l|}{ Laboratory results } \\
\hline White blood cells (mean $\pm \mathrm{SD}$ ), $\times 10^{9} / \mathrm{L}$ & $11.6 \pm 5.1$ & $10.2 \pm 3.7$ & $0.50^{\mathrm{a}}$ \\
\hline Hemoglobin $($ mean $\pm \mathrm{SD}), \mathrm{g} / \mathrm{dL}$ & $9.1 \pm 1.5$ & $8.3 \pm 2.1$ & $0.32^{\mathrm{a}}$ \\
\hline Hematocrit (mean $\pm \mathrm{SD}), \%$ & $26.3 \pm 4.6$ & $24.6 \pm 5.9$ & $0.48^{\mathrm{a}}$ \\
\hline Platelet count (median, IQR), $\times 10^{9} / \mathrm{L}$ & $14.1(8.8-27.9)$ & $11.5(6.4-19.9)$ & $0.22^{\mathrm{a}}$ \\
\hline $\mathrm{LDH}($ mean $\pm \mathrm{SD}), \mathrm{IU} / \mathrm{L}$ & $838.1 \pm 365.4$ & $1,103.1 \pm 546.5$ & $0.23^{\mathrm{a}}$ \\
\hline Creatinine (median, IQR), mg/dL & $0.9(0.9-4.3)$ & $1.6(1.5-1.8)$ & $0.39^{\mathrm{c}}$ \\
\hline PT (median, IQR), s & $15.2(13.9-16.9)$ & $14.8(13.9-16.0)$ & $0.68^{\mathrm{c}}$ \\
\hline $\mathrm{PTT}($ mean $\pm \mathrm{SD}), \mathrm{s}$ & $33.7 \pm 8.0$ & $29.9 \pm 4.0$ & $0.21^{\mathrm{a}}$ \\
\hline Fibrinogen $($ mean $\pm S D), \mathrm{mg} / \mathrm{dL}$ & $431.2 \pm 100.8$ & $405.1 \pm 129.5$ & $0.67^{\mathrm{a}}$ \\
\hline D-dimer (median, IQR), ng/mL $(n=16)$ & $708.0(460.5-5,966.0)$ & $2,155.0(1,768.3-6,485.3)$ & $0.07^{\mathrm{c}}$ \\
\hline Troponin (median, (IQR), ng/mL & $1.1(0.4-11.3)$ & $0.42(0.25-1.16)$ & $1.00^{\mathrm{c}}$ \\
\hline ADAMTS13 activity, U/dL & $<5$ & $<5$ & n.d. \\
\hline Anti-ADAMTS13 IgG $($ mean \pm SD $), \mathrm{U} / \mathrm{mL}(n=17)$ & $6,750.1 \pm 4,737.7$ & $5,485.6 \pm 1,924.7$ & $0.99^{\mathrm{a}}$ \\
\hline Inhibitor (median, IQR), IU $(n=15)$ & $3.2(0.7-6.0)$ & $0.8(0.5-1.6)$ & $0.12^{\mathrm{c}}$ \\
\hline Citrullinated $\mathrm{H}_{3}$ (median, IQR), ng/mL $(n=15)$ & $3.2(2.6-3.5)$ & $7.1(4.2-9.7)$ & $0.009^{c}$ \\
\hline Histone/DNA complex (mean $\pm \mathrm{SD}), \mathrm{U} / \mathrm{mL}(n=18)$ & $60.3 \pm 46.0$ & $165.8 \pm 94.2$ & $0.014^{\mathrm{a}}$ \\
\hline Cell-free DNA (median, IQR), ng/mL $(n=18)$ & $863.3(753.6-1,143.6)$ & $1,076.7(894.5-1,580.2)$ & $0.30^{c}$ \\
\hline
\end{tabular}

$N$, total number of patients with imaging; $n$, number of patients tested; $\mathrm{LDH}$, lactate dehydrogenase; PT, prothrombin time; PTT, activated thromboplastin time; SD, standard deviation; IQR, interquartile range; n.d., not determined. ${ }^{a} t$ test. ${ }^{b} \chi^{2}$ test. ${ }^{\mathrm{c}}$ Mann-Whitney $\mathrm{U}$ test. Italicized $p$ values are statistically significant.

strokes had higher risk of exacerbation, but it did not reach statistical significance (HR 2.38, 95\% CI 0.54-13.1; $p=0.23$; Table 4 ). See Figure 1 for an example of an iTTP patient with a large ischemic stroke.

\section{Discussion}

In this study of a large cohort of consecutive patients with acute iTTP, we found that many of these patients with neurologic symptoms who had a stroke suffered from large ischemic strokes as opposed to the classically described small ischemic strokes associated with iTTP. Patients with larger strokes attributed to iTTP tended to be younger than patients with the classic small strokes on presentation. This highlights that iTTP is a potential stroke etiology to consider for patients with unknown causes of large and small ischemic strokes with otherwise no significant vascular risk factors and lack of atherosclerosis of the large vessels on their vessel imaging. In our population, there were no differences between size of stroke and recurrence of iTTP or mortality rates. The early diagnosis of iTTP as a potential etiology of large ischemic strokes could potentially be a reason for this lack of difference though our sample size was small.

Nevertheless, our study is the largest cohort study so far in the literature to analyze neuroimaging in patients with stroke caused by iTTP. Most prior studies have 
been either case reports or small series [14, 28, 30, 31]. The largest neuroimaging review of iTTP patients performed by Burrus et al. [26] screened over 200 patients to identify 47 patients with neuroimaging available. Of those, 5 patients had stroke on CT scan, and 10 patients had small ischemic stroke on MRI. In our cohort, we were able to identify 65 patients with neurological symptoms, of which 21 had reviewable neuroimaging that demonstrated stroke. Fifty-two percent $(n=11)$ had what are considered large ischemic strokes while $48 \%(n=10)$ had the classic small ischemic stroke associated with iTTP. Interestingly, Burrus et al. [26] did not report any large ischemic strokes in their cohort. Previous literature reviews have only identified a total of 10 reported cases of strokes in TTP caused by large ischemic strokes [14]. This is the first systematic study to identify that large ischemic strokes can be common in iTTP patients with stroke. Having a large ischemic stroke on neuroimaging should not eliminate the possibility of iTTP being an etiology.

Prior studies have not consistently reported their TTP-associated laboratory values. By not reporting the level of ADAMTS13, we do not know how many of these cases were in fact having a diagnosis of TTP [14]. The large neuroimaging cohort from Burrus et al. [26] did not report their laboratory values either. Therefore, they could not associate the large ischemic stroke presentations to the TTP severity. While we did not find any difference in plasma levels of ADAMTS13 activity, ADAMTS13 inhibitor, and anti-ADAMTS13 IgG between the large and small ischemic stroke groups, this is quite expected as these parameters are critical for establishing a diagnosis of iTTP. Interestingly, the ADAMTS13 inhibitor levels appeared to be borderline higher in the large stroke group.

Intriguingly, we did find a significant difference between the 2 groups in both citrullinated histone $\mathrm{H}_{3}$ and the histone-DNA complex levels. Patients with large ischemic strokes had lower levels of citrullinated histone $\mathrm{H}_{3}(3.0 \mathrm{vs.} 7.8 \mathrm{ng} / \mathrm{mL})$ and lower levels of histoneDNA complex (60.3 vs. $165.8 \mathrm{U} / \mathrm{mL}$ ) than patients with small ischemic stroke. Citrullinated histone $\mathrm{H}_{3}$ and histone-DNA complex are parts of neutrophil extracellular traps (NETs), released from acute inflammation and neutrophil apoptosis and necrosis. These NETs are formed after citrullination of histones by activation of protein arginine deiminase 4 , which has been associated with thrombosis in patients with cancers [40, 41]. Leukocytes are known to release nuclear contents that form these extracellular traps, which are mainly com- posed of the histone-DNA complex [42]. These biomarker levels that lead to extracellular trap formation have been shown to be higher in serum circulation in patients who receive peripheral angioplasty with stent implementation that then develop strokes [43] and higher in stroke patients with internal artery occlusions [44]. Our data are the first to report these biomarkers are also associated with iTTP-induced stroke. Interestingly, the small ischemic stroke had higher levels of the NET biomarkers (i.e., citrullinated histone $\mathrm{H}_{3}$ and histone-DNA complex, etc.). Since leukocytic levels are increased from an inflammatory response, it is possible that iTTP may be triggered by and/or leads to a significant inflammatory response, which results in a secondary microscopic polyangiitis, a vasculitis of small ischemic disease, in addition to thrombosis. Further research is needed to classify whether there are different mechanisms of stroke etiology in patients with iTTPinduced stroke and the role of NET biomarkers.

Similar to other retrospective cohort analyses, our study has several limitations. While neuroimaging was obtained for neurological symptom evaluation, exact timing of the examinations was variable ranging from hours to a week. Unfortunately, since these patients presented medically ill, the clinical severity of the stroke was not routinely recorded in these patients. They were often admitted to nonstroke units and so an NIH Stroke Scale was not obtained. This was a registry collected from a single center, though the patients come from across multiple states. Since many of these acute patients presenting with iTTP have other causes for neurological disorders and potential neuroimaging changes, we could not perform a more detailed analysis of potential confounders, including TOAST stroke subtype criteria and location of the small strokes, because of the relatively limited sample size.

We conclude that iTTP-induced stroke patients can commonly present with large ischemic strokes. These patients tend to be younger than cases with the classically associated small ischemic strokes. Further research should be performed in assessing different etiologies of iTTP-induced stroke based on NET formation biomarkers seen in small ischemic stroke. Targeted therapeutics aiming at eliminating NETs such as the use of DNase I may be considered in preclinical animal models and in iTTP patients with stroke to reduce neurocognitive sequelae.
Cerebrovasc Dis Extra 2021;11:29-36 DOI: $10.1159 / 000513574$
Lin/Memon/Sui/Zheng 


\section{Statement of Ethics}

The present study conforms to the guidelines issued in the Declaration of Helsinki. This study was approved by the Institutional Review Committee (100811004). Subjects (or their parents or guardians) have given their written informed consent.

\section{Conflict of Interest Statement}

X.L.Z. is a speaker or consultant for Alexion, Sanofi, and Takeda. X.L.Z. is also the Founder of Clotsolution Inc. All other authors have declared no relevant conflict. All other authors report no conflicts of interest concerning the materials or methods used in this study or the findings specified in this paper.

\section{Funding Sources}

C.L. is supported by a Veterans Affairs Research Development Award. The study is supported in part by grants from NHLBI (HL126724) and Answering TTP foundation (awarded to X.L.Z.).

\section{Author Contributions}

C.L., R.M., J.S., and X.L.Z. designed research, performed experiments, and analyzed the results, as well as wrote the manuscript. All other nonauthors in the acknowledgement contributed to patient recruitment, informed consents, and sample collections. All authors have reviewed and approved the final version of the paper for submission.

\section{References}

1 Tsai HM. Pathophysiology of thrombotic thrombocytopenic purpura. Int J Hematol. 2010 Jan;91(1):1-19.

2 Bell WR, Braine HG, Ness PM, Kickler TS. Improved survival in thrombotic thrombocytopenic purpura-hemolytic uremic syndrome. Clinical experience in 108 patients. $\mathrm{N}$ Engl J Med. 1991 Aug;325(6):398-403.

3 Terrell DR, Williams LA, Vesely SK, Lämmle B, Hovinga JA, George JN. The incidence of thrombotic thrombocytopenic purpura-hemolytic uremic syndrome: all patients, idiopathic patients, and patients with severe ADAMTS-13 deficiency. J Thromb Haemost. 2005 Jul;3(7):1432-6.

4 Tsai HM, Lian EC. Antibodies to von Willebrand factor-cleaving protease in acute thrombotic thrombocytopenic purpura. N Engl J Med. 1998 Nov;339(22):1585-94.

5 Zheng XL, Wu HM, Shang D, Falls E, Skipwith CG, Cataland SR, et al. Multiple domains of ADAMTS13 are targeted by autoantibodies against ADAMTS13 in patients with acquired idiopathic thrombotic thrombocytopenic purpura. Haematologica. 2010 Sep;95(9): 1555-62.

6 Levy GG, Nichols WC, Lian EC, Foroud T, McClintick JN, McGee BM, et al. Mutations in a member of the ADAMTS gene family cause thrombotic thrombocytopenic purpura. Nature. 2001 Oct;413(6855):488-94.

7 Kokame K, Matsumoto M, Soejima K, Yagi H, Ishizashi $\mathrm{H}$, Funato $\mathrm{M}$, et al. Mutations and common polymorphisms in ADAMTS13 gene responsible for von Willebrand factorcleaving protease activity. Proc Natl Acad Sci USA. 2002 Sep;99(18):11902-7.

8 Zheng XL, Sadler JE. Pathogenesis of thrombotic microangiopathies. Annu Rev Pathol. 2008;3(1):249-77.
9 Dong JF, Moake JL, Nolasco L, Bernardo A, Arceneaux W, Shrimpton CN, et al. ADAMTS-13 rapidly cleaves newly secreted ultralarge von Willebrand factor multimers on the endothelial surface under flowing conditions. Blood. 2002 Dec;100(12):4033-9.

10 Dong JF, Moake JL, Bernardo A, Fujikawa K, Ball C, Nolasco L, et al. ADAMTS-13 metalloprotease interacts with the endothelial cellderived ultra-large von Willebrand factor. J Biol Chem. 2003 Aug;278(32):29633-9.

11 Zheng XL. ADAMTS13 and von Willebrand factor in thrombotic thrombocytopenic purpura. Annu Rev Med. 2015;66(1):211-25.

12 Laje P, Shang D, Cao W, Niiya M, Endo M, Radu A, et al. Correction of murine ADAMTS13 deficiency by hematopoietic progenitor cell-mediated gene therapy. Blood. 2009 Mar;113(10):2172-80.

13 Niiya M, Endo M, Shang D, Zoltick PW, Muvarak NE, Cao W, et al. Correction of ADAMTS13 deficiency by in utero gene transfer of lentiviral vector encoding $\mathrm{AD}$ AMTS13 genes. Mol Ther. 2009 Jan;17(1): 34-41.

14 Sugarman R, Tufano AM, Liu JM. Large vessel stroke as initial presentation of thrombotic thrombocytopenic purpura. BMJ Case Rep. 2018 Feb 5;2018:bcr2017221857.

15 Chauhan AK, Kisucka J, Brill A, Walsh MT, Scheiflinger F, Wagner DD. ADAMTS13: a new link between thrombosis and inflammation. J Exp Med. 2008 Sep;205(9):2065-74.

16 Chauhan AK, Motto DG, Lamb CB, Bergmeier W, Dockal M, Plaimauer B, et al. Systemic antithrombotic effects of ADAMTS13. J Exp Med. 2006 Mar;203(3):767-76.

17 Rock GA, Shumak KH, Buskard NA, Blanchette VS, Kelton JG, Nair RC, et al.; Canadian Apheresis Study Group. Comparison of plasma exchange with plasma infusion in the treatment of thrombotic thrombocytopenic purpura. N Engl J Med. 1991 Aug;325(6): 393-7.
18 Scully M, McDonald V, Cavenagh J, Hunt BJ, Longair I, Cohen $\mathrm{H}$, et al. A phase 2 study of the safety and efficacy of rituximab with plasma exchange in acute acquired thrombotic thrombocytopenic purpura. Blood. 2011 Aug; 118(7):1746-53.

19 Zheng X, Pallera AM, Goodnough LT, Sadler JE, Blinder MA. Remission of chronic thrombotic thrombocytopenic purpura after treatment with cyclophosphamide and rituximab. Ann Intern Med. 2003 Jan;138(2):105-8.

20 Saha M, McDaniel JK, Zheng XL. Thrombotic thrombocytopenic purpura: pathogenesis, diagnosis and potential novel therapeutics. J Thromb Haemost. 2017 Oct;15(10):1889900.

21 Peyvandi F, Scully M, Kremer Hovinga JA, Cataland S, Knöbl P, Wu H, et al.; TITAN Investigators. Caplacizumab for Acquired Thrombotic Thrombocytopenic Purpura. N Engl J Med. 2016 Feb;374(6):511-22.

22 Scully M, Cataland SR, Peyvandi F, Coppo P, Knöbl P, Kremer Hovinga JA, et al.; HERCULES Investigators. Caplacizumab Treatment for Acquired Thrombotic Thrombocytopenic Purpura. N Engl J Med. 2019 Jan;380(4):33546.

23 Zheng XL, Vesely SK, Cataland SR, Coppo P, Geldziler B, Iorio A, et al. ISTH guidelines for the diagnosis of thrombotic thrombocytopenic purpura. J Thromb Haemost. 2020 Oct; 18(10):2486-95.

24 Moschcowitz E. An acute febrile pleiochromic anemia with hyaline thrombosis of the terminal arterioles and capillaries; an undescribed disease. Am J Med. 1952 Nov;13(5): 567-9.

25 Meloni G, Proia A, Antonini G, De Lena C, Guerrisi V, Capria S, et al. Thrombotic thrombocytopenic purpura: prospective neurologic, neuroimaging and neurophysiologic evaluation. Haematologica. 2001 Nov;86(11):11949. 
26 Burrus TM, Wijdicks EF, Rabinstein AA. Brain lesions are most often reversible in acute thrombotic thrombocytopenic purpura. Neurology. 2009 Jul;73(1):66-70.

27 Sadler JE. What's new in the diagnosis and pathophysiology of thrombotic thrombocytopenic purpura. Hematology (Am Soc Hematol Educ Program). 2015;2015(1):631-6.

28 Rinkel GJ, Wijdicks EF, Hené RJ. Stroke in relapsing thrombotic thrombocytopenic purpura. Stroke. 1991 Aug;22(8):1087-9.

29 Scheid R, Hegenbart U, Ballaschke O, Von Cramon DY. Major stroke in thromboticthrombocytopenic purpura (Moschcowitz syndrome). Cerebrovasc Dis. 2004;18(1):835.

30 Boattini M, Procaccianti G. Stroke due to typical thrombotic thrombocytopenic purpura treated successfully with intravenous thrombolysis and therapeutic plasma exchange. BMJ Case Rep. 2013 Jan;2013:bcr2012008426.

31 Yu WL, Leung T, Soo Y, Lee J, Wong KS. Thrombotic thrombocytopenic purpura with concomitant small- and large-vessel thrombosis, atypical posterior reversible encephalopathy syndrome and cerebral microbleeds. Oxf Med Case Rep. 2015 Feb;2015(2):179-82.

32 Staley EM, Cao W, Pham HP, Kim CH, Kocher NK, Zheng L, et al. Clinical factors and biomarkers predict outcome in patients with immune-mediated thrombotic thrombocytopenic purpura. Haematologica. 2019 Jan; 104(1):166-75.
33 Sui J, Cao W, Halkidis K, Abdelgawwad MS, Kocher NK, Guillory B, et al. Longitudinal assessments of plasma ADAMTS13 biomarkers predict recurrence of immune thrombotic thrombocytopenic purpura. Blood Adv. 2019 Dec;3(24):4177-86.

34 Wardlaw JM, Smith EE, Biessels GJ, Cordonnier C, Fazekas F, Frayne R, et al.; STandards for ReportIng Vascular changes on nEuroimaging (STRIVE v1). Neuroimaging standards for research into small vessel disease and its contribution to ageing and neurodegeneration. Lancet Neurol. 2013 Aug;12(8):822-38.

35 Lin C, Sangha R, Lee J, Corado C, Jalasutram A, Chatterjee N, et al. Infarct location is associated with quality of life after mild ischemic stroke. Int J Stroke. 2018 Oct;13(8):824-31.

36 Lin C, Chatterjee N, Lee J, Harvey R, Prabhakaran S. Predictive value of the combination of lesion location and volume of ischemic infarction with rehabilitation outcomes. Neuroradiology. 2019 Oct;61(10):1131-6.

37 Goldstein LB, Jones MR, Matchar DB, Edwards LJ, Hoff J, Chilukuri V, et al. Improving the reliability of stroke subgroup classification using the Trial of ORG 10172 in Acute Stroke Treatment (TOAST) criteria. Stroke. 2001 May;32(5):1091-8.

38 Lin C, Babiker A, Srdanovic N, Kocherginsky M, Harvey RL. Depressive symptoms after stroke are associated with worse recovery. Int J Psychiatry Med. 2020 Jul;55(4):227-38.

39 Lin C, Arevalo YA, Nanavati HD, Lin DM. Racial differences and an increased systemic inflammatory response are seen in patients with COVID-19 and ischemic stroke. Brain Behav Immun Health. 2020 Oct;8:100137.
40 Mauracher LM, Posch F, Martinod K, Grilz E, Däullary T, Hell L, et al. Citrullinated histone H3, a biomarker of neutrophil extracellular trap formation, predicts the risk of venous thromboembolism in cancer patients. J Thromb Haemost. 2018 Mar;16(3):508-18.

41 Grilz E, Mauracher LM, Posch F, Königsbrügge $\mathrm{O}$, Zöchbauer-Müller S, Marosi C, et al. Citrullinated histone $\mathrm{H} 3$, a biomarker for neutrophil extracellular trap formation, predicts the risk of mortality in patients with cancer. Br J Haematol. 2019 Jul;186(2):311-20.

42 Yoo HJ, Lee JS, Kim JE, Gu J, Koh Y, Kim I, et al. Extracellular Histone Released from Leukemic Cells Increases Their Adhesion to Endothelium and Protects them from Spontaneous and Chemotherapy-Induced Leukemic Cell Death. PLoS One. 2016 Oct; 11(10):e0163982

43 Demyanets S, Stojkovic S, Mauracher LM, Kopp CW, Wojta J, Thaler J, et al. Surrogate Markers of Neutrophil Extracellular Trap Formation are Associated with Ischemic Outcomes and Platelet Activation after Peripheral Angioplasty and Stenting. J Clin Med. 2020 Jan; $9(2):$ :E304.

44 Zhou P, Li T, Jin J, Liu Y, Li B, Sun Q, et al Interactions between neutrophil extracellular traps and activated platelets enhance procoagulant activity in acute stroke patients with ICA occlusion. EBioMedicine. 2020 Mar;53: 102671. 